University of Wollongong

Research Online

Faculty of Engineering and Information

Faculty of Engineering and Information

Sciences - Papers: Part A

Sciences

$1-1-2014$

\title{
Enhanced wall clutter mitigation for compressed through-the-wall radar imaging using joint Bayesian sparse signal recovery
}

Van Ha Tang

University of Wollongong, vht986@uowmail.edu.au

Abdesselam Bouzerdoum

University of Wollongong, bouzer@uow.edu.au

Son Lam Phung

University of Wollongong, phung@uow.edu.au

Fok Hing Chi Tivive

University of Wollongong, tivive@uow.edu.au

Follow this and additional works at: https://ro.uow.edu.au/eispapers

Part of the Engineering Commons, and the Science and Technology Studies Commons

Research Online is the open access institutional repository for the University of Wollongong. For further information contact the UOW Library: research-pubs@uow.edu.au 


\title{
Enhanced wall clutter mitigation for compressed through-the-wall radar imaging using joint Bayesian sparse signal recovery
}

\author{
Abstract \\ This paper addresses the problem of wall clutter mitigation in compressed sensing through-the-wall radar \\ imaging, where a different set of frequencies is sensed at different antenna locations. A joint Bayesian \\ sparse approximation framework is first employed to reconstruct all the signals simultaneously by \\ exploiting signal sparsity and correlations between antenna signals. This is in contrast to previous \\ approaches where the signal at each antenna location is reconstructed independently. Furthermore, to \\ promote sparsity and improve signal reconstruction accuracy, a sparsifying wavelet dictionary is \\ employed in the sparse signal recovery. Following signal reconstruction, a subspace projection technique \\ is applied to remove wall clutter, prior to image formation. Experimental results on real data show that the \\ proposed approach produces significantly higher reconstruction accuracy and requires far fewer \\ measurements compared to the single-signal model, where each antenna signal is reconstructed \\ independently.

\section{Keywords} \\ mitigation, clutter, wall, enhanced, sparse, bayesian, joint, imaging, recovery, radar, signal, compressed \\ Disciplines \\ Engineering | Science and Technology Studies

\section{Publication Details} \\ V. Tang, A. Bouzerdoum, S. Phung \& F. Tivive, "Enhanced wall clutter mitigation for compressed through- \\ the-wall radar imaging using joint Bayesian sparse signal recovery," in IEEE International Conference on \\ Acoustics, Speech and Signal Processing (ICASSP 2014), 2014, pp. 7804-7808.
}




\title{
ENHANCED WALL CLUTTER MITIGATION FOR COMPRESSED THROUGH-THE-WALL RADAR IMAGING USING JOINT BAYESIAN SPARSE SIGNAL RECOVERY
}

\author{
V. H. Tang, A. Bouzerdoum, S. L. Phung, and F. H. C. Tivive \\ School of Electrical, Computer and Telecommunications Engineering, \\ University of Wollongong, NSW, 2522, Australia
}

\begin{abstract}
This paper addresses the problem of wall clutter mitigation in compressed sensing through-the-wall radar imaging, where a different set of frequencies is sensed at different antenna locations. A joint Bayesian sparse approximation framework is first employed to reconstruct all the signals simultaneously by exploiting signal sparsity and correlations between antenna signals. This is in contrast to previous approaches where the signal at each antenna location is reconstructed independently. Furthermore, to promote sparsity and improve reconstruction accuracy, a sparsifying wavelet dictionary is employed in the sparse signal recovery. Following signal reconstruction, a subspace projection technique is applied to remove wall clutter, prior to image formation. Experimental results on real data show that the proposed approach produces significantly higher reconstruction accuracy and requires far fewer measurements for forming high-quality images, compared to the single-signal compressed sensing model, where each antenna signal is reconstructed independently.
\end{abstract}

Index Terms - Through-the-wall radar imaging, wall clutter mitigation, compressed sensing, joint Bayesian sparse signal recovery.

\section{INTRODUCTION}

Through-the-wall radar imaging (TWRI) is an emerging technology that has attracted considerable research interest due to its numerous civilian and military applications [1-3]. In TWRI, the scene behind the wall is first interrogated by transmitting wideband electromagnetic (EM) waves; then, the reflected signals from the wall and targets are processed to form the image. For target detection and localization, the clutter caused by the front wall EM returns must be mitigated prior to image formation since strong wall reflections obscure stationary targets, especially those with small radar-cross-section.

Typically, the scene image is formed by backprojection methods, such as delay-and-sum (DS) beamforming [1]. Recently, by exploring the sparsity of signals, compressed sensing (CS) has been applied for fast data acquisition and effi-

This work was partially supported by a grant from the Australian Research Council. cient signal reconstruction $[4,5]$. The first attempts of employing CS for TWRI [6-10] assume that the wall returns can be completely removed before applying CS, or a background scene is available for suppressing the wall reflections. Very recently, wall mitigation techniques were investigated in conjunction with CS [11]. If the same frequency measurements are available at each antenna, spatial filtering [12] or subspace projection $[13,14]$ methods can be applied directly for wall clutter removal. However, having the same frequency observations is not possible due to competing wireless services or intentional interferences [15].

In general compressed TWRI, different frequency measurements are collected at different antennas. Hence, the missing frequency measurements need to be recovered before applying either spatial filtering or subspace methods because the phase returns vary along the antenna locations, rendering the application of such wall mitigation techniques ineffective. In [11], CS is applied for signal recovery at each antenna separately. However, the images formed by this single-signal CS model are degraded when the measurements are drastically decreased. One of the major reasons is that the signal sparsity is reduced due to wall returns. Moreover, recovering signal independently requires more measurements from each antenna. Insufficient target signals in the measurement sets lead to a weak CS constraint for signal recovery.

In this paper, we propose a new approach for enhancing wall clutter mitigation and compressed TWRI. A joint Bayesian sparse approximation framework is first employed for reconstructing all the signals along the antenna array simultaneously, by considering both signal sparsity and correlations. Because of the wall returns and target reflections, the sparsity of signals, in terms of range profiles, is reduced. To overcome this issue, a sparsifying wavelet dictionary is incorporated into the model. Under the wavelet representation, the significant wavelet coefficients across the antenna locations appear at almost the same positions (i.e. same sparsity support). Hence, in the proposed model, the signals are correlated, and their correlations should be exploited for improving signal reconstruction and reducing the compressive measurements. Then, wall mitigation techniques, such as subspace projection, are applied on the recovered signals for removing the wall returns, followed by image formation. 
The remainder of the paper is organized as follows. Section 2 reviews the through-the-wall radar signal model. Section 3 presents the single-signal CS recovery model for wall mitigation and compressed TWRI. Section 4 describes the proposed approach, formulating a linear model for joint Bayesian sparse signal reconstruction in compressed TWRI. Section 5 presents experimental results and analysis. Section 6 gives concluding remarks.

\section{THROUGH-THE-WALL RADAR SIGNAL MODEL}

Consider a monostatic stepped-frequency TWRI system that uses $M$ antenna locations and $N$ narrowband signals to image a scene containing $P$ targets placed behind a homogeneous wall of thickness $d$ and dielectric constant $\epsilon$. Let $z_{m, n}$ denote the signal of frequency $f_{n}$ received at the $m$-th antenna location. The signal $z_{m, n}$ can be expressed as

$$
z_{m, n}=\sigma_{w} \exp \left(-j 2 \pi f_{n} \tau_{m, w}\right)+\sum_{p=0}^{P-1} \sigma_{p} \exp \left(-j 2 \pi f_{n} \tau_{m, p}\right)
$$

where $\sigma_{w}$ and $\sigma_{p}$ are, respectively, the complex reflectivities of the wall and the $p$-th target, $\tau_{m, w}$ and $\tau_{m, p}$ are the roundtrip travel times of the signal from the $m$-th antenna location to the wall and the $p$-th target, respectively.

Assume that the scene is partitioned into a rectangular grid. Using DS beamforming [1], a complex-valued image is formed by aggregating the measurements $z_{m, n}$. The value of the pixel at coordinates $(x, y)$ is given by

$$
I(x, y)=\frac{1}{M N} \sum_{m=0}^{M-1} \sum_{n=0}^{N-1} z_{m, n} \exp \left(j 2 \pi f_{n} \tau_{m,(x, y)}\right)
$$

where $\tau_{m,(x, y)}$ is the focusing delay between the $m$-th transceiver and the target located at the pixel position $(x, y)$.

To reveal the targets, wall reflections must be removed from the received signals before image formation. If the full data volume is available, wall mitigation techniques [12-14] can be applied directly. However, for practical compressed TWRI, we have only reduced data sets acquired along the antennas. Hence, the missing frequency samples at each antenna position need to be recovered before applying wall clutter mitigation techniques.

\section{SINGLE-SIGNAL CS MODEL}

Let $\mathbf{z}_{m}=\left[z_{0, m}, \ldots, z_{N-1, m}\right]^{T}$ be the received signal at the $m$-th antenna location. Suppose that the range profile $\mathbf{u}_{m}$ is partitioned into $N$ equidistant cells $u_{m, i}$, where $i=0,1, \ldots, N-1$. Let $\tau_{m, i}$ be the two-way signal travel time between the $m$-th antenna and the $i$-th range cell. The
$N$-dimensional range vector $\mathbf{u}_{m}$ is defined as

$$
u_{m, i}= \begin{cases}\sigma_{w}, & \text { if } \tau_{m, i}=\tau_{m, w} \\ \sigma_{p}, & \text { if } \tau_{m, i}=\tau_{m, p} \\ 0, & \text { otherwise. }\end{cases}
$$

Then, the signal is related to the target location as follows:

$$
\mathbf{z}_{m}=\Psi \mathbf{u}_{m}, \text { for } m=0,1, \ldots, M-1,
$$

where $\boldsymbol{\Psi}$ is an $N \times N$ matrix, $[\boldsymbol{\Psi}]_{n i}=\exp \left(-j 2 \pi f_{n} \tau_{i}\right)$. In compressed TWRI, the reduced frequency observations $\mathbf{y}_{m}$ collected at each antenna can be expressed as $\mathbf{y}_{m}=\boldsymbol{\Phi}_{m} \mathbf{z}_{m}$, where $\boldsymbol{\Phi}_{m}$ is a $K_{m} \times N$ selection matrix $\left(K_{m}<N\right)$ containing a single non-zero element in each row and each column. From Eq. (4), it follows that

$$
\mathbf{y}_{m}=\boldsymbol{\Phi}_{m} \Psi \mathbf{u}_{m}=\mathbf{D}_{m} \mathbf{u}_{m}
$$

where $\mathbf{D}_{m}=\boldsymbol{\Phi}_{m} \boldsymbol{\Psi}$ is a $K_{m} \times N$ dictionary matrix. Let $\varepsilon$ be a noise bound. The range profile $\mathbf{u}_{m}$ can be recovered by solving the following CS inverse problems:

$$
\hat{\mathbf{u}}_{m}=\arg \min _{\mathbf{u}_{m}}\left\|\mathbf{u}_{m}\right\|_{1} \text { s. t. }\left\|\mathbf{D}_{m} \mathbf{u}_{m}-\mathbf{y}_{m}\right\|_{2} \leq \varepsilon
$$

In $[7,11]$, the signals were recovered independently, one at a time. This recovery scheme is known as single-signal CS technique. After recovering all the signals, a wall clutter mitigation technique is applied to remove the wall returns from the target signal.

\section{JOINT BAYESIAN SPARSE SIGNAL MODEL}

Because of the wall returns and target reflections, the sparsity of the range profile $\mathbf{u}_{m}$ is reduced, rendering the CS application ineffective. A sparsifying wavelet dictionary $\mathbf{W}$ is therefore incorporated into the model to represent the range vector,

$$
\mathbf{u}_{m}=\mathbf{W} \boldsymbol{\theta}_{m}
$$

where $\boldsymbol{\theta}_{m}$ is a vector of wavelet coefficients. Substituting Eq. (7) into Eq. (5), we obtain

$$
\mathbf{y}_{m}=\mathbf{D}_{m} \mathbf{W} \boldsymbol{\theta}_{m}=\widetilde{\mathbf{D}}_{m} \boldsymbol{\theta}_{m}
$$

where $\widetilde{\mathbf{D}}_{m}=\mathbf{D}_{m} \mathbf{W}$. To account for noise in the radar signal, the measurement vector $\mathbf{y}_{m}$ is modeled as:

$$
\mathbf{y}_{m}=\widetilde{\mathbf{D}}_{m} \boldsymbol{\theta}_{m}+\mathbf{v}_{m},
$$

where $\mathbf{v}_{m}$ is a zero-mean white Gaussian noise vector.

The problem in Eq. (9) is considered as a joint sparsity model. That is the supports of the wavelet coefficient vectors $\left\{\boldsymbol{\theta}_{m}\right\}_{m=0}^{M-1}$ are assumed to have considerable overlap. As an example, Fig. 1 shows the wavelet coefficients computed using real TWRI signals (see Section 5 for the radar 


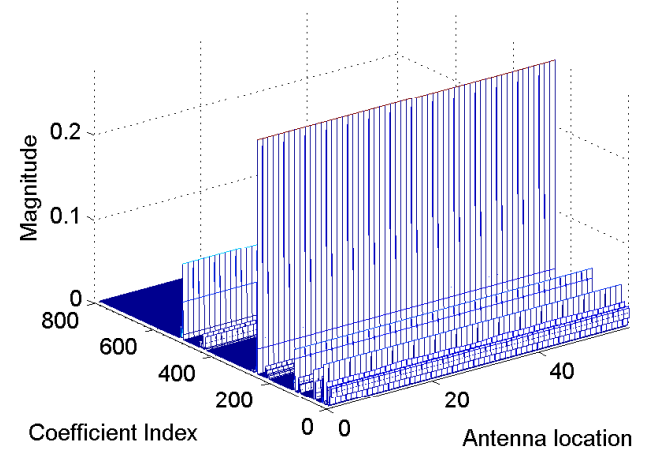

Fig. 1. Wavelet coefficients $\boldsymbol{\theta}_{m}$ at all antenna locations computed from the full measurements set.

system setup). It is observed that the significant wavelet coefficients along the antenna locations have a common sparsity support. Therefore, the reconstruction accuracy can be improved significantly by taking into account the signal correlations among the antennas.

Several joint or simultaneous sparse approximation algorithms have been proposed which exploit correlations between signals [16-18]. However, these methods are not suitable for the TWRI problem since they assume all measurement sets are obtained using the same sensing matrix, which in TWRI context corresponds to the constraint of having the same frequency bins at different antenna locations. To relax this constraint, the joint Bayesian sparse signal recovery framework is employed for jointly estimating the wavelet coefficients $\boldsymbol{\theta}_{m}$ [19]. This approach models the likelihood of the vector $\boldsymbol{\theta}_{m}$ as a multivariate Gaussian function,

$$
p\left(\mathbf{y}_{m} \mid \boldsymbol{\theta}_{m}, \beta\right)=(2 \pi / \beta)^{-K_{m} / 2} \exp \left(-\frac{\beta}{2}\left\|\mathbf{y}_{m}-\widetilde{\mathbf{D}}_{m} \boldsymbol{\theta}_{m}\right\|^{2}\right),
$$

where $\beta$ is the noise precision. The signal sparsity is guaranteed via a shared prior imposed on $\boldsymbol{\theta}_{m}$,

$$
p\left(\boldsymbol{\theta}_{m} \mid \boldsymbol{\alpha}, \beta\right)=\prod_{n=0}^{N-1} \mathcal{N}\left(\theta_{m, n} \mid 0, \beta^{-1}, \alpha_{n}^{-1}\right)
$$

Given the hyper-parameters $\boldsymbol{\alpha}=\left\{\alpha_{0}, \alpha_{1}, \ldots, \alpha_{N-1}\right\}$, by Bayes' rule, the posterior density function for $\boldsymbol{\theta}_{m}$ is a multivariate Student-t distribution with the following mean and covariance [19]:

$$
\begin{aligned}
\boldsymbol{\mu}_{m} & =\Sigma_{m} \widetilde{\mathbf{D}}_{m}^{T} \mathbf{y}_{m} \\
\Sigma_{m} & =\left(\widetilde{\mathbf{D}}_{m}^{T} \widetilde{\mathbf{D}}_{m}+\mathbf{A}\right)^{-1}
\end{aligned}
$$

where $\mathbf{A}=\operatorname{diag}\left(\alpha_{0}, \alpha_{1}, \ldots, \alpha_{N-1}\right)$. The problem now becomes searching for the hyper-parameters $\boldsymbol{\alpha}$ by maximizing the marginal likelihood, or equivalently its logarithm,

$$
\begin{gathered}
\hat{\boldsymbol{\alpha}}=\arg \max _{\boldsymbol{\alpha}} \mathcal{L}(\boldsymbol{\alpha}), \\
\mathcal{L}(\boldsymbol{\alpha})=\sum_{m=0}^{M-1} \log p\left(\mathbf{y}_{m} \mid \boldsymbol{\alpha}\right) .
\end{gathered}
$$

The optimization problem in Eq. (14) can be solved efficiently using a fast iterative algorithm. Note that all measurement sets $\left\{\mathbf{y}_{m}\right\}_{m=0}^{M-1}$ are used for the hyper-parameter estimation via the summation over the conditional distributions in Eq. (15). Hence, the signal correlations across the antennas are exploited through the estimation of the hyper-parameters. The reader is referred to $[19,20]$ for a detailed description of the algorithm. Once the hyper-parameters $\boldsymbol{\alpha}$ are estimated, the wavelet coefficients $\boldsymbol{\theta}_{m}$ are obtained by the mean of the posterior given in Eq. (12). Then, the range profiles and the full frequency measurements are recovered using Eqs. (7) and (4), respectively.

\section{EXPERIMENTAL RESULTS AND ANALYSIS}

The proposed approach is evaluated on real radar data acquired with a radar system placed in front of a concrete wall of thickness $0.143 \mathrm{~m}$, and dielectric constant $\epsilon=7.6$. A stepped-frequency signal between 0.7 and $3.1 \mathrm{GHz}$, with $3 \mathrm{MHz}$ frequency step, was used to scan a scene containing a $0.4 \mathrm{~m}$ high and $0.3 \mathrm{~m}$ wide dihedral. A 57-element line array with an inter-element spacing of $0.022 \mathrm{~m}$ was placed at a standoff distance of $1.016 \mathrm{~m}$ away from the wall. The imaged scene, extending from $[0,4] \mathrm{m}$ in downrange and $[-2,2] \mathrm{m}$ in crossrange, was partitioned into an image of size $96 \times 96$ pixels.

To solve the single-signal CS recovery problems in Eq. (6), convex relaxation basis pursuit denoising (BPDN) [21] and greedy orthogonal matching pursuit (OMP) [22] were used. The noise bound $\varepsilon$ was estimated using a crossvalidation strategy described in [23]. For sparsifying signals, the wavelet dictionary $\mathbf{W}$ was constructed using Daubechies wavelets of order 4 . The normalized mean squared error (NMSE) was used to measure the reconstruction error:

$$
\mathrm{NMSE}=\|\mathbf{z}-\hat{\mathbf{z}}\|_{2} /\|\mathbf{z}\|_{2},
$$

where $\hat{\mathbf{z}}$ and $\mathbf{z}$ are the reconstructed and true signals, respectively. The image quality was measured using the target-toclutter ratio (TCR) [24] (in dB):

$$
\mathrm{TCR}=10 \log _{10}\left(P_{\text {target }} / P_{\text {clutter }}\right)
$$

where $P_{\text {target }}$ and $P_{\text {clutter }}$ are the average power of the target and clutter regions, respectively.

In the first experiment, only $20 \%$ of the full frequency observations were used. Figure 2 shows the signals at the center antenna location recovered by different approaches. 


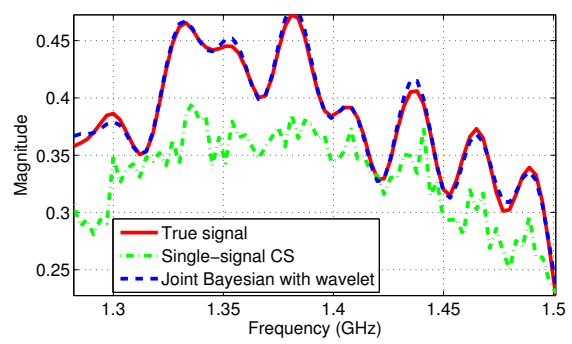

Fig. 2. Reconstructed antenna signal using single-signal CS with BPDN (dotted line), and joint Bayesian sparse signal model with wavelet dictionary (dashed line).

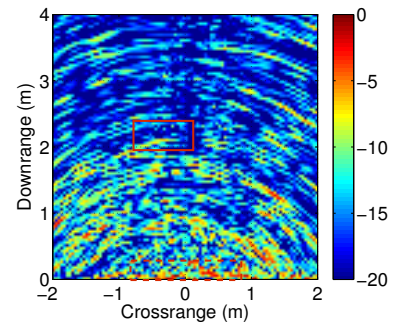

(a)

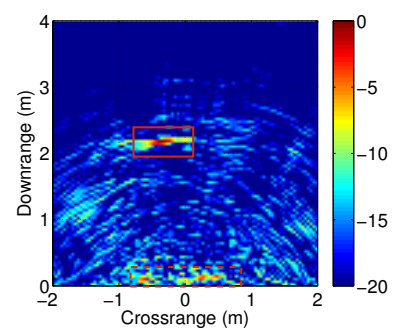

(c)

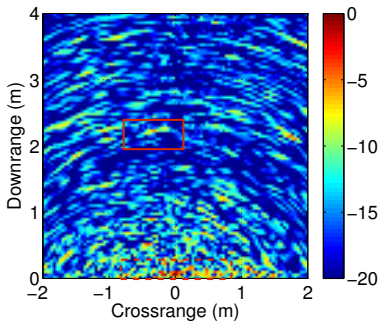

(b)

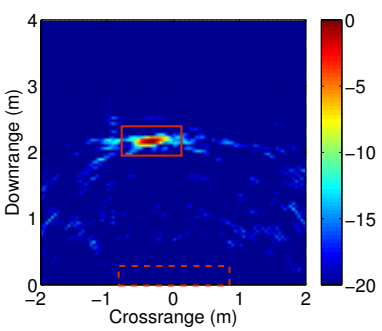

(d)
Fig. 3. Image of a single target formed using $20 \%$ frequency measurements; (a) single-signal CS with BPDN (TCR $=-0.843 \mathrm{~dB})$; (b) single-signal $\mathrm{CS}$ with OMP (TCR $=-0.129 \mathrm{~dB}$ ); (c) joint Bayesian sparse signal recovery (TCR $=29.567 \mathrm{~dB})$; (d) joint Bayesian sparse signal recovery with wavelet dictionary $(\mathrm{TCR}=41.960 \mathrm{~dB})$.

It is observed that compared to the single-signal CS recovery, the joint Bayesian sparse approach produces a significantly lower reconstruction error. The subspace projection method [14] was then applied to the reconstructed signals for wall clutter mitigation before forming the image of the scene. Figures 3(a)-(b) show the images formed after the singlesignal CS recovery and wall removal. It is observed that the images are degraded, containing a large amount of clutter; the target and wall clutter regions are indicated by solid and dashed rectangles, respectively. Figures 3(c)-(d) present the images reconstructed with the joint Bayesian sparse model and wall clutter mitigation. The target is well localized with high intensity values. Figure 3(d) shows that incorporating a sparsifying wavelet dictionary further enhances the perfor-

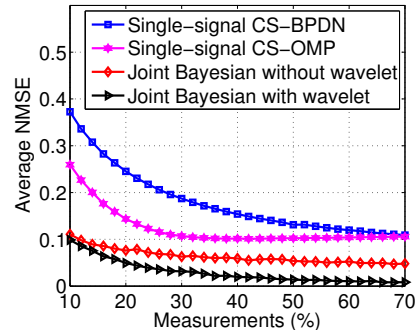

(a)

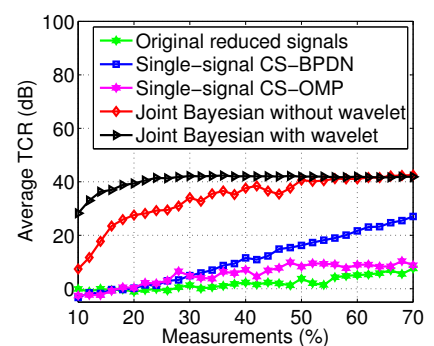

(b)
Fig. 4. Performances of different imaging approaches: (a) average NMSE of the reconstructed signals; (b) average TCR of the reconstructed images.

mance: the wall clutter and background noise are significantly attenuated.

We then varied the size of the compressive frequency measurements from $10 \%$ to $70 \%$. For each set of measurements, the experiment was repeated 30 times and the average NMSE and TCR were recorded. Figure 4 illustrates the NMSE and TCR as a function of the number of measurements. Figure 4(a) shows that compared to the single-signal CS recovery model, the joint Bayesian sparse approach produces a considerably lower reconstruction error. This observation is consistent for all the measurements. Moreover, to obtain the same reconstruction accuracy, the proposed approach requires far fewer measurements than does its singlesignal CS counterpart. For example, to obtain a NMSE $=0.1$, the joint Bayesian sparse approach requires only $10 \%$ measurements, whereas the single-signal CS model uses $30 \%$. The superiority of the reconstruction by the joint Bayesian sparse signal model is due to the fact that this approach exploits the signal sparsity as well as the interdependencies between signals. Figure 4(b) demonstrates that incorporating wavelet representation yields more stable image recovery and enhances the image quality.

\section{CONCLUSION}

We have presented a new approach for through-the-wall radar imaging, which incorporates wall clutter mitigation, using a joint Bayesian compressive sensing framework. First a joint Bayesian sparse approximation is proposed for simultaneous signal reconstruction from the reduced set of measurements where the sparsity and correlations between antenna signals are exploited. A sparsifying wavelet dictionary is incorporated to promote signal sparsity and improve signal reconstruction accuracy. Second, a subspace projection technique is applied to the recovered signals to segregate wall clutter from the target signal prior to image formation. Experimental results on real data demonstrate the superiority of the proposed approach over the single-signal CS model. 


\section{REFERENCES}

[1] F. Ahmad, M. G. Amin, and S. A. Kassam, "Synthetic aperture beamformer for imaging through a dielectric wall," IEEE Trans. Aerospace and Electronic Systems, vol. 41, no. 1, pp. 271-283, 2005.

[2] M. G. Amin (Ed.), Through-The-Wall Radar Imaging. Boca Raton, FL: CRC Press, 2010.

[3] C. H. Seng, A. Bouzerdoum, M. G. Amin, and S. L. Phung, "Probabilistic fuzzy image fusion approach for radar through wall sensing," IEEE Trans. Image Processing, vol. 22, no. 12, pp. 4938-4951, 2013.

[4] D. L. Donoho, "Compressed sensing," IEEE Trans. Information Theory, vol. 52, no. 4, pp. 1289-1306, 2006.

[5] E. J. Candes, J. Romberg, and T. Tao, "Stable signal recovery from incomplete and inaccurate measurements," Communications on Pure and Applied Mathematics, vol. 59, no. 8, pp. 1207-1223, 2006.

[6] Q. Huang, L. Qu, B. Wu, and G. Fang, "UWB throughwall imaging based on compressive sensing," IEEE Trans. Geoscience and Remote Sensing, vol. 48, no. 3, pp. 1408-1415, 2010.

[7] Y.-S. Yoon and M. G. Amin, "Through-the-wall radar imaging using compressive sensing along temporal frequency domain," in Proc. IEEE Int. Conf. Acoustics, Speech, and Signal Processing, 2010, pp. 2806-2809.

[8] M. Leigsnering, C. Debes, and A. M. Zoubir, "Compressive sensing in through-the-wall radar imaging," in Proc. IEEE Int. Conf. Acoustics, Speech, and Signal Processing, 2011, pp. 4008-4011.

[9] J. Yang, A. Bouzerdoum, F. H. C. Tivive, and M. G. Amin, "Multiple-measurement vector model and its application to through-the-wall radar imaging," in Proc. IEEE Int. Conf. Acoustics, Speech and Signal Processing, 2011, pp. 2672-2675.

[10] V. H. Tang, A. Bouzerdoum, and S. L. Phung, "Twostage through-the-wall radar image formation using compressive sensing," Journal of Electronic Imaging, vol. 22, no. 2, pp. 021 006-021 006, 2013.

[11] E. Lagunas, M. G. Amin, F. Ahmad, and M. Njar, "Joint wall mitigation and compressive sensing for indoor image reconstruction," IEEE Trans. Geoscience and Remote Sensing, vol. 51, no. 2, pp. 891 - 906, 2013.

[12] Y.-S. Yoon and M. G. Amin, "Spatial filtering for wallclutter mitigation in through-the-wall radar imaging," IEEE Trans. Geoscience and Remote Sensing, vol. 47, no. 9, pp. 3192-3208, 2009.

[13] F. H. C. Tivive, A. Bouzerdoum, and M. G. Amin, "An SVD-based approach for mitigating wall reflections in through-the-wall radar imaging," in Proc. IEEE Radar Conf., 2011, pp. 519-524.

[14] F. H. C. Tivive and A. Bouzerdoum, "An improved SVD-based wall clutter mitigation method for throughthe-wall radar imaging," in Proc. IEEE Workshop on Signal Processing Advances in Wireless Communications, 2013, pp. 430-434.

[15] F. Ahmad and M. G. Amin, "Partially sparse reconstruction of behind-the-wall scenes," in Proc. SPIE compressive sensing $I$, vol. 8365, 2012, pp. $83650 \mathrm{~W}-83650 \mathrm{~W}-$ 9.

[16] J. A. Tropp, A. C. Gilbert, and M. J. Strauss, "Algorithms for simultaneous sparse approximation. part I: Greedy pursuit," Signal Processing, vol. 86, no. 3, pp. 572-588, 2006.

[17] S. F. Cotter, B. D. Rao, K. Engan, and K. KreutzDelgado, "Sparse solutions to linear inverse problems with multiple measurement vectors," IEEE Trans. Signal Processing, vol. 53, no. 7, pp. 2477-2488, 2005.

[18] J. A. Tropp, "Algorithms for simultaneous sparse approximation. part II: Convex relaxation," Signal Processing, vol. 86, no. 3, pp. 589-602, 2006.

[19] S. Ji, D. Dunson, and L. Carin, "Multitask compressive sensing," IEEE Trans. Signal Processing, vol. 57, no. 1, pp. 92-106, 2009.

[20] V. H. Tang, A. Bouzerdoum, S. L. Phung, and F. H. C. Tivive, "Enhanced through-the-wall radar imaging using Bayesian compressive sensing," in Proc. SPIE compressive sensing II, vol. 8717, 2013, pp. $87170 \mathrm{I}-$ 87 170I-12.

[21] S. S. Chen, D. L. Donoho, and M. A. Saunders, "Atomic decomposition by basis pursuit," SIAM Journal on Scientific Computing, vol. 20, pp. 33-61, 1999.

[22] J. A. Tropp and A. C. Gilbert, "Signal recovery from random measurements via orthogonal matching pursuit," IEEE Trans. Information Theory, vol. 53, no. 12, pp. 4655-4666, 2007.

[23] A. C. Gurbuz, J. H. McClellan, and W. R. Scott, "A compressive sensing data acquisition and imaging method for stepped frequency GPRs," IEEE Trans. Signal Processing, vol. 57, no. 7, pp. 2640-2650, 2009.

[24] C. H. Seng, A. Bouzerdoum, M. G. Amin, and S. L. Phung, "Two-stage fuzzy fusion with applications to through-the-wall radar imaging," IEEE Geoscience and Remote Sensing Letters, vol. 10, no. 4, pp. 687-691, 2012. 